\title{
RECENSIÓN
}

\section{CONOCIMIENTOS ACTUALES SOBRE NUTRICIÓN}

Ekhard E. Ziegler, L.J. Filer, editores. Séptima edición.

Organización Panamericana de la Salud-Instituto Internacional de Ciencias de la Vida. Publicación Científica N. ${ }^{\circ} 565$.

Año 1997.

ISBN 9275315655.

731 páginas.

Recensión: Fernando Villar Alvarez. Subdirección General de Epidemiología, Promoción y Educación para la Salud. Dirección General de Salud Pública. Ministerio de Sanidad y Consumo.

Acaba de aparecer la séptima edición en español de la clásica publicación Conocimientos actuales sobre nutrición, que supone un gran esfuerzo de actualización de la creciente base científica de la nutrición y de sus aplicaciones a otras disciplinas relacionadas. Se trata de un libro fruto de la colaboración entre la Organización Panamericana de la Salud y el Instituto Internacional de Ciencias de la Vida (International Life Science Institute, ILSI), del que han sido editores los doctores Ekhard E. Ziegler y L.J. Filer, del Departamento de Pediatría de la Facultad de Medicina de la Universidad de Iowa. Los autores de los distintos capítulos son destacados expertos de la nutrición, que trabajan en su mayoría en los Estados Unidos.

El libro, magníficamente traducido, constituye un amplio tratado sobre la nutrición. Consta de 64 capítulos (13 de ellos nuevos respecto a la edición anterior), que a lo largo de 731 páginas desarrolla de forma exhaustiva y actual, como los propios cditores dicen, con el ánimo de informar y estimular, los distintos aspectos de la nutrición. Los principios inmediatos, vitaminas y minerales se detallan pormenorizadamente en los capítulos iniciales. También se abordan los aspectos nutricionales en grupos o situaciones especiales, como el embarazo y lactancia, en los lactantes, los adolescentes y en el envcjccimicnto. En la relación entrc la alimentación y las enfermedades se analiza la aterosclerosis, la hipertensión arterial, el cáncer, la diabctes, la osteoporosis y osteomalacia, las enfermedades renales, hepáticas y gastrointestinales. Hay capítulos específicos para el alcohol, la obesidad y el ejercicio físico. Se dedican algunos capítulos al cálculo de la ingesta alimentaria, la epidemiología de la nutrición, la vigilancia de la nutrición y las pautas y guías de alimentos. En definitiva, un completo tratado sobre la nutrición.

Todos los capítulos proporcionan una idea clara y precisa del conocimiento actual de cada tema, fundamentado en una bibliografía completa y seleccionada.

Llama la atención el escaso espacio dedicado a los trastornos del comportamiento alimentario y, en particular, a la anorexia y la bulimia, de creciente importancia por afectar al bienestar físico y psíquico de muchos adolescentes. Quizá en la próxima edición puedan ser tratados estos temas con mayor profundidad. 
Esta publicación puede resultar de gran interés como libro de texto o de consulta, para los nutricionistas, los clínicos, los investigadores, el personal de los servicios de salud, los docentes y los estudiantes de ciencias de la salud que quieran profundizar en los distintos aspectos del campo de la nutrición. Como ejemplo de su amplia difusión y utilización baste decir que las ediciones anteriores fueron traducidas a seis idiomas, entre ellas el español.
Dentro del espléndido esfuerzo editorial de la Organización Panamericana de la Salud, también ha publicado otros libros de gran interés sobre nutrición en lengua española. Entre ellos se puede mencionar el Primer informe sobre la situación de la nutrición en el mundo; Guía para evaluar el estado de nutrición de Beghin, Cap y Dujardin; Unidades didácticas de nutrición; Vigilancia alimentaria y nutricional en las Américas. 